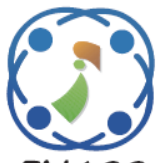

\title{
Vortex Search Topology Control Algorithm for Wireless Sensor Networks
}

\author{
Essam Halim Houssein ${ }^{1 *} \quad$ Yaser Maher Wazery $^{1}$ \\ ${ }^{1}$ Faculty of Computers and Information, Minia University, Egypt \\ * Corresponding author's Email: essam.halim@mu.edu.eg
}

\begin{abstract}
Wireless sensor networks (WSNs) is composed of a huge number of sensor nodes that are densely deployed in a target environment. One of the major fundamental challenges in WSNs is the Topology control (TC). TC algorithms try to reduction the average of nodes transition radius without reduction of the network connectivity. This paper has proposed a metaheuristic algorithm for TC based on the vortex search (VS) algorithm for WSNs termed VSTC. VSTC algorithm dynamically adjusts transition radius of nodes, so the proper transition radius can be obtained using VS algorithm, so the position of each node is to calculate and represented in a binary format to enhance the coverage area and reduce the number of active nodes. Furthermore, the proposed algorithm is under a less average number of neighbours and the energy consumption compared with the present algorithms. In addition, the proposed algorithm is simulated with the A1 topology algorithm (A1), A3 topology algorithm (A3), and topology control scheme based on particle swarm optimization algorithm (PSOTCS) algorithm, the experimental results have been provided the highest in terms of the high residual energy by $34 \%$ and the number of active nodes was decreased by $18 \%$.
\end{abstract}

Keywords: Wireless sensor networks (WSNs), Topology control (TC), Meta-heuristic, Vortex search (VS) algorithm, Energy consumption, A1 topology algorithm, A3 topology algorithm.

\section{Introduction}

A Wireless sensor network consisting of a huge number distributed sensors deployed in an ad hoc fashion that communicates by means of wireless technology and organizes themselves in a multi-hop communication wireless network. The sensor nodes have the capabilities of sensing, processing, communicating and they operate in an unattended environment, with limited computational and sensing capabilities [1-2]. Energy in WSN is thus a scarce and precious resource that should be saved [3]. WSNs are becoming a rapidly developing area in both research and application. WSNs have been envisioned for a wide range of applications, such as battlefield intelligence, environmental tracking, and emergency response. Each sensor node has the limited computational capacity, battery supply, and communication capability [4-6].

Topology control is one of the major fundamental problems in WSNs. It is an efficient factor to ensure the quality of connectivity and coverage because it determines how to maintain network connectivity and transmit the power of each node while consuming as minimum power as possible [7-8]. Without convenient TC algorithms in placing a randomly connected multi-hop wireless sensor network may susceptible to trivial network utilization, high end-to-end delays, and shortened network lifetime. In general, the TC can be divided into three types. The first type is to reduce the node redundancy in a network that usually periodically let picked nodes enter an energy-saving mode. The second type is to use the clustering strategy which utilized a rotation of the cluster head to evenly distribute the energy consumption. The third type concentrates on reducing link redundancy. The proposed algorithm in this paper would be applied to find out the solution for the TC scheme in WSNs deal with the first and the third TC type.

The metaheuristic categorization, which distinguishes between the single-based metaheuristic and the population-based metaheuristic. Single- 
based metaheuristics, which are based on a single solution at any time and include local search-based metaheuristics such as simulated annealing (SA), Iterated local search (ILS), and random search (RS). Though, in population-based metaheuristics, a number of solutions are first created and then updated iteratively until the termination condition is satisfied. Population-based metaheuristics are generally studied under two major groups: Evolutionary algorithms and swarm-based algorithms [9]. Both classifications have been successfully utilized to many real-world optimization problems. These algorithms return solutions by exploring the search space efficiently while reducing the effective size of the search. Thus, the achievement of a metaheuristic method on a particular optimization problem is defined by its ability to provide a good balance between the exploration and exploitation. The exploration realizes the global search ability of the algorithm, while the exploitation is the ability to locate the optimum round a near-optimal solution, which can also be rate as the local search ability. In this paper, we will utilize the vortex search (VS) algorithm [10], a new single-based metaheuristic, to obtain the best construction TC for WSN.

As aforementioned, the WSNs have a dynamic topology and need to adjust a huge number of sensors, the algorithms, and algorithms designed should be distributed and localized to better adapt their scalable architecture. Therefore, in this paper, we will propose a metaheuristic-based topology control algorithm for WSNs based on vortex search algorithm termed VSTC. The TC algorithms classify the topology as a connected network and locate the set of active nodes. TC algorithms use node IDs of different nodes and a node selection criteria for nodes to calculate their timeout. In this way, a reduced topology is formed while keeping the network connected and covered. To achieve energy efficiency, VSTC comprising of high energy nodes in a single-phase construction process. In addition, it also forms a proportionate set of active nodes to provide better sensing coverage. Moreover, we compare the performance of VSTC algorithm with A1 algorithm [11], A3 algorithm [12] and PSOTCS algorithm [13]. In the same context, we perform comprehensive simulations under varying network sizes to achieve the effectiveness of VSTC. The experimental results demonstrated that VSTC algorithm has low message complexity, provides better residual energy resources while having less number of unconnected nodes when compared with the other three algorithms.
The rest of this paper is organized as follows. Section 2 summarizes the literature review in this area. In Section 3, we introduce a review of preliminaries used in this paper. We explain the proposed VSTC algorithm in Section 4. Section 5 dedicated to the experimental results and shows the discussion on simulation results. Finally, we summarize the salient findings of this paper and give some perspectives in Section 6 .

\section{Literature review}

In recent years, a lot of many types of research have been achieved to overcome the limitations of WSNs, to remedy the design and application issues. The features of sensor networks and application requirements have a direct impact on the network design issues in terms of network performance and capabilities. Due to huge sensor nodes and the dynamics of their operating environment, these were considered as unique challenges on the architectural design of WSNs. Many researchers have approached TC problem from different perspectives in WSNs. Here we classified it into two types due to the techniques have been used to solve the TC problem.

\subsection{Swarm-based}

Swarm intelligence plays a critical role in adaptive TC and an efficient random sensor deployment scheme is very important due to the similarity of the nature of Swarm intelligence and WSNs manner.

Based on glowworm swarm optimization (GSO), Liao et al. [14], have introduced a sensor deployment scheme based on GSO to enhance the coverage after an initial random deployment. Each sensor node is considered as individual glowworms and the intensity of the luciferin is following the distance between the sensor node and its neighbouring sensors. Simulation results show that GSO-based sensor deployment approach can achieve high coverage with restricted movement of the sensor nodes.

Furthermore, some studies based on particle swarm optimization (PSO) to enhancing the TC problem. Robert and José [15], have studied the minimum energy network connectivity (MENC) problem, which minimizes the energy consumption of the network while keeping its global connectivity. They have proposed a heuristic algorithm based on the PSO to obtain near-optimal solutions. The proposed heuristic is tested on a set of 50 instances of the problem. The computational results show that heuristic algorithm performs better than the classical minimum spanning tree (MST) heuristic. Nikdel et 
al., [16], have proposed a particle swarm optimization topology control algorithm for WSNs called PSOTC dynamically adjusts transition radius of nodes and to determine the proper transition radius using PSO algorithm. PSOTC algorithm has a less average number of neighbours compared to the existing algorithms. Also, the energy consumption in this algorithm is less than other algorithms and the network lifetime will be extended. In addition, the PSOTC algorithm is simulated and its performance is demonstrated by the simulation results.

Furthermore, Guo et al., [17], have presented a PSO-optimized minimum spanning tree-based topology control scheme to overcome high connectivity redundancy and low structure robustness in traditional methods. Furthermore, TC scheme based is proposed. Simulation results show that the proposed topology can converge to the nondominated front quite evenly, and the topology derived has lower total power consumption, higher robust structure, and lower contention among nodes. Wenzhong et al., [18], have presented the problem of TC in WSNs. Taking energy consumption and reliability into account and consider $\mathrm{TC}$ to be a trouble of multi-objective degree-constrained minimum spanning tree (mcd-MST). Also, they have presented an improved discrete particle swarm optimization (PSO) algorithm for generating such topology schemes. The simulation results show that this algorithm could obtain higher robust structure and lower contention topology schemes compared with genetic algorithm (GA). Also, Goyal et al., [19], have applied a meta-heuristic optimization algorithm using bat algorithm to evaluate the precision of node localization problem in WSNs. Compared with the existing bat algorithm, the proposed modified bat algorithm is shown through simulations to perform constantly better not only in increasing localization success ratios and fast convergence speed but also enhance its robustness.

\subsection{Location-based}

In addition to the above, numerous approaches are introduced for coordinate estimation of the sensor nodes. The main aim of these methods is to estimate the coordinates of the sensor nodes with a weak error. Zhang et al., [20], have proposed two location-assisted grid-based topology control (GBP) algorithms. The design objective of GBP algorithm is to effectively reduce the number of active nodes required to keep global network connectivity and divide the network in grid-based topology control, into two spaced squares. Nedal [21], has presented a
TC algorithm for WSNs which uses two-hop neighbourhood information to select a subset of nodes to be active among all nodes in the neighbourhood. Each node in the network selects its own set of active neighbours from among its onehop neighbours. This set is determined such that it covers all two-hop neighbours. The proposed algorithm is evaluated against two well-known algorithms from the literature through realistic simulations using TOSSIM. Konstantinidis et al., [22], proposed a memetic algorithm based solution for energy-aware topology control for WSNs termed ToCMA, utilizing a combination of problemspecific light-weighted local search and genetic algorithms. Outcomes of ToCMA can be used for different network optimization and fault-tolerant purposes. Also, Aloor and Jacob [23], have proposed a computationally efficient distributed WSN localization method based on Stochastic Proximity Embedding (SPE), which is a dimensionality reduction technique that finds a lowdimensional embedding of a high dimensional data by preserving the pairwise distance data information. Also, the stochastic descent approach adopted in SPE provides an accurate position estimate in a reasonable number of iterations. Meanwhile inclusive simulation study of the suggested method, it is found to provide better results in both uniform and irregularly shaped sensor networks. Finally, Jianping et al., [24], have presented an approach for the WSN to maximize the topological network lifetime, based on an algorithm in computational Geometry. In addition, they have established the upper and lower bounds of their maximal topological lifetime. The experimental performance evaluation demonstrates the efficacy of TC as a vital process to maximize the network lifetime of WSNs.

\section{Preliminaries}

In this section, we describe the algorithms and techniques we will use in this paper.

\subsection{Topology control algorithm}

Topology control algorithm is a process of controlling the number of active links between nodes or selecting a minimum subset of nodes to join the working backbone, to reduce the energy consumption within WSNs. The major challenge for these algorithms is how to achieve this energy conservation without sacrificing the important network's characteristics such as connectivity and sensing coverage [25]. It is worth pointing out, TC algorithms categories are not unique as some algorithms can be classified into different categories. 
Therefore, if the focus and perspective are about the transition radius, TC can be classified as homogeneous and heterogeneous. In the homogeneous TC, all network nodes utilize the same transition radius and TC problem is to locate a minimum value for transition radius considering the network features such as network connectivity and coverage. Regarding the heterogeneous TC, the network nodes cannot have regular transition radius [26].

On the other hand, if the emphasis is placed on the cover area, TC can be classified as deterministic deployment [27] and random deployment [28]. In deterministic deployment, the sensor nodes are circulated in dedicated locations so that the service area is quite covered and the network connectivity is maintained. This style of deployment can exactly compatible with the user's requirements, but it needs former information of deployment environment, which is not always available. In addition, deterministic deployment is not always feasible as the sensor nodes are used to monitor and study the places that are unreachable or dangerous for human presence. While random deployment is more flexible as the sensor nodes can be put conveniently by dissemination and without previously information about the surveillance environment. This style of deployment though easy to implement and convenient in all kinds of environment, cannot always guarantee the complete coverage and connectivity of the service region [29].

The major purpose of a TC algorithms in WSNs is to reduce node power consumption in order to enhance the network lifetime. These studies [30-32] present a comprehensive overview of TC algorithms proposed in the literature for WSNs. TC can be considered as a complete directed graph $\mathrm{G}=(\mathrm{V}, \mathrm{E})$, where $\mathrm{n}=|\mathrm{V}|, \mathrm{V}$ is a finite set of vertices representing terminals, E finite set of edges, each edge has $\mathrm{m}$ weights, and the problem is to find all minimum spanning tree (MST). In most of TC algorithms, the coordinate of vertices in $\mathrm{G}$ and the weights of each edge are generated randomly. Fig. 1 shows the initial topology generated using 50 vertices.

Choosing appropriate topology for WSN has so much effect on networks' performance, especially considering power consumption and a lifetime of the network.

\subsection{A1 topology algorithm}

Rizvi, Sajjad, et al., [11], have presented an energy efficient reduced topology. The fundamental

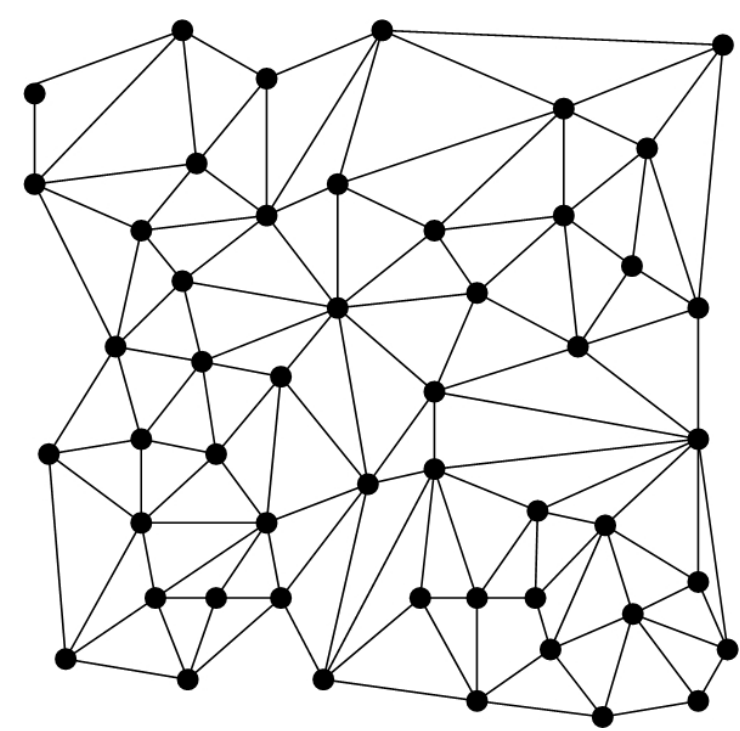

Figure.1 Initial network topologies of 50 vertices

design application that has used to reduce the size of the backbone nodes is with the help of signal strength and energy based timeout criteria. The nodes chosen criteria for timeout is given by the following formula:

$$
T_{d, s}=\left(\frac{E_{d}}{E_{i}}\right)+\left(\frac{R S S_{s}}{R S S_{c}}\right)
$$

Where $\mathrm{d}$ and $\mathrm{s}$ represent the children node and parent node, $\mathrm{E}_{\mathrm{d}}$ is the residual energy level of the children node and $E_{i}$ is the primary energy level. Similarly, $\mathrm{RSS}_{\mathrm{s}}$ is the signal strength of parent node received by the children node and $\mathrm{RSS}_{\mathrm{c}}$ is the lower required signal strength to guarantee connectivity. The selection criteria allow high energy nodes with the better signal strength to be selected. This is due to the reason that the neighbours of the node select a low value for timeout if they calculate a high value for selection criteria. The selected nodes serve as a virtual backbone for all the nodes in the network and hence forming a CDS. The A1 constructs the topology in one phase. At the initial, the initiator node first finds out its neighbour. Similarly, the neighbours of the initiator node find out their neighbours as their timeout expires in the second phase. This process continues until the complete topology is created with nodes acting as the virtual backbone (CDS) for rest of the nodes in the network.

\subsection{A3 topology algorithm}

Wightman and Labrador [12], have introduced A3 topology, which addresses the problem of finding a reduced topology. A3 detects a suboptimal connected dominating Set (CDS) to turn 


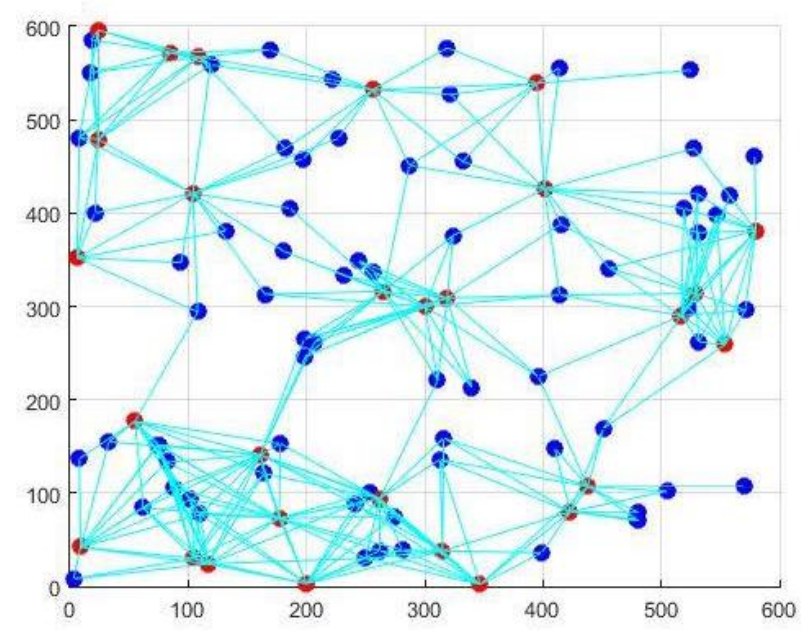

Figure.2 The A3 Algorithm

dispensable nodes off while keeping the network connected and covered. The algorithm is based on a rising tree technique and uses a selection metric based on the residual energy of the nodes and distance between them. The choosing metric allows the network operator to pick a more reliable shortlived network with a larger number of nodes and a less reliable longer-lasting tree with fewer nodes. furthermore, the A3 algorithm introduces the following advantages: a) A3 is extremely scalable, as it only needs local information and operates in a completely distributed manner; b) A3 does not need location information; no any localization mechanism is needed; c) A3 requires no synchronization scheme; d) A3 is easy and presents low computational complexity, and e) A3 is efficient in term of energy. First, the CDS tree building process is done in only one phase and the node selection process avoids the use of node competition or twohop information queries, which reduces the overhead. Second, the small number of active nodes after the topology construction minimizes the number of collisions considerably. Finally, A3 has a low and linearly bounded message complexity, which allows the algorithm to be run many times as part of the TC iterative cycle with very low energy cost.

The A3 algorithm supposes no previous knowledge about the position or orientation of the nodes; subsequently, the nodes do not have an accurate geometric view of the topology. However, nodes can locate how far a node is based on the strength of the signal received, and this information is enough to select a close-to-optimal CDS tree, based on the confidence that further nodes will offer a better area of communication coverage. The A3 algorithm is executed in three processes; neighbourhood discovery, children selection, and second opportunity as depicted in Fig. 2 for 100 nodes network size.

The evaluation performance of the A3 algorithm compared with two existing TC algorithms is studied. In terms of the number of active nodes needed to build the reduced topology, the A3 algorithm performs better than the compared algorithms as demonstrated from the results.

\subsection{PSOTCS algorithm}

Bingyu et al. [13], have proposed new swarmbased topology control called PSOTCS and evaluate the performance of PSOTCS against SMECN and LMST with respect to several metrics via simulation, and all the TC algorithms are implemented in MATLAB. The authors make the distance between each pair of nodes, the coverage of an edge, and the residual energy of nodes as the foundation of setting up the structure. The authors have adopted the idea of LMST algorithm, transform the model into a problem of degree-constrained minimum spanning tree and design a discrete Particle Swarm Optimization algorithm to deal with this issue. The results of PSOTCS algorithm are compared to those of SMECN and LMST to demonstrate the effectiveness of the proposed methodology and shows that PSOTCS can provide a network topology with low power consumed, low radio contention and high robust structure, and it is easy to be implemented.

PSOTCS run through four processes; 1) representation of particles, 2) fitness value function, 3) the parameter settings of DPSO and 4) discrete procedure of PSO. More overview about the PSOTCS algorithm is presented here in [13]. From our standpoint, they used a number of sensor nodes in the region from 40 to 80 , this range is considered a shortage to prove the effectiveness of the PSOTCS algorithm. In contrast in our proposed VSTC algorithm, we have used more than 2000 sensor nodes to evaluate the effectiveness of VSTC over the existing TC algorithms.

\subsection{Vortex search algorithm (VS)}

Doğan and Ölmez have proposed a new singlesolution based metaheuristic, namely, the vortex search (VS) algorithm [10], for the solution of bound constrained global optimization problems. VS algorithm have been studied within the family of the search algorithms that comprise the Random Search and Pattern Search algorithms. Also, VS algorithm uses a new adaptive step size adjustment scheme that considerably improves the performance of the 
search process. Generation of candidate solutions by using some neighborhood structures is of critical importance for the success of the single-solution based metaheuristics. In single-solution based methods one of the main properties searched for a neighborhood is the locality. When small changes are made on the current solution, the neighborhood is said to have a strong locality. In contrast, a weak locality is characterized by a large effect on the solution, which results in the search being a random search in the search space. VS algorithm consist of four main processes as follows; 1) generating the initial solution, 2) generating the candidate solutions, 3) replacement of the current solution and 4) the radius decrement process.

In the selection phase of the second process, the new set of solutions is evaluated to select a solution. If the selected solution is better than the best solution found so far, then this solution is assigned to be the new best solution and it is memorized. Next, the center of the third circle is assigned to be the memorized best solution found so far. This process iterates until the termination condition is met. In this manner, once the algorithm is terminated, the resulting pattern appears as a vortexlike structure, where the center of the smallest circle is the optimum point found by the algorithm. From our standpoint, VS algorithm is suitable for TC in WSN depending on the nature work of the algorithm.

\section{The proposed VSTC algorithm}

The network lifetime points out to how long the deployed WSN can do their responsibility well. It can be defined as the period between the time when the network was set up and the time when the WSN cannot guarantee determined coverage or connectivity requirements. The network lifetime can be extended by finding several subsets of sensors and schedule them to transmit data to the sink. As a result, power consumption balance is accomplished by sensor deployment scheme.

The sink node, which is situated at the center of layer 0 in WSN, collects the sensing information from other sensors. The sensors in the vicinity of the sink susceptible to consuming more energy than those ultimate away from the sink. This is fundamental because of the fact that besides transmitting their own packets, they also further transmit packets on behalf of last sensors that have resided away. We assume that there are $\mathrm{N}$ sensor nodes to be deployed in the service region and at least one sensor must be deployed in the established in advance circle points. One of the sensors in each circle point is chosen as the sensor head, which wariness of the movement of the sensors in its circle point to extend the network lifetime. The sensor heads gather up information on remaining and consumed the energy of all the sensor in their circle point and interchange this information with their neighboring sensor heads periodically. This paper aims to propose a mechanism to construct an efficient TC to move the sensors to convenient circle points in order to extend the lifetime of the WSN called VSTC algorithm based on enhanced VS algorithm.

In VSTC algorithm and similar to VS algorithm, generating the initial solution and consider a twodimensional optimization problem. In a twodimensional space, a vortex pattern can be represented by a number of nested circles. Here, the outer circle of the vortex is first centered on the search space, where the initial center $\mu_{0}$ can be figured using Eq. (2).

$$
\mu_{0}=\frac{\text { upperlimit }+ \text { lowerlimit }}{2}
$$

Where upperlimit and lowerlimit are $\mathrm{dx} 1$ vectors that define the bound constraints of the problem in $\mathrm{d}$ dimensional space.

A number of neighbour solutions $\mathrm{C}_{\mathrm{t}}(\mathrm{s})$, ( $\mathrm{t}$ describes the iteration index and initially $\mathrm{t}=0$ ) are randomly created around the initial center $\mu_{0}$ in the two-dimensional space by using a Gaussian distribution. Here, $\mathrm{C} 0(\mathrm{~s})=\{\mathrm{s} 1, \mathrm{~s} 2, \ldots, \mathrm{sk}\}, \mathrm{k}=1$, $2, \ldots, \mathrm{n}$ describes the solutions, and $\mathrm{n}$ characterize the total number of candidate solutions. In Eq. (3), the general form of the multivariate Gaussian distribution is stated.

$$
p(x \mid \mu, \Sigma)=\frac{1}{\sqrt{(2 \pi)^{d}|\Sigma|}} \exp \left\{-\frac{1}{2}(x-\mu)^{T} \Sigma^{-1}(x-\mu)\right\}
$$

where $\mathrm{d}$ describes the dimension, $\mathrm{x}$ is the $\mathrm{dx} 1$ vector of a random variable, $\mu$ is the $d x 1$ vector of sample mean and $\Sigma$ is the covariance matrix. If the diagonal elements of the values of $\Sigma$ are equal and if the off-diagonal elements are zero, then the generating shape of the distribution will be spherical in which can be considered circular for a twodimensional problem. Thus, the value of $\Sigma$ can be figured by using equal variances with zero covariance by using Eq. (4).

$$
\Sigma=\sigma^{2} \cdot[I]_{d x d}
$$


In Eq. (3), $\sigma^{2}$ descibes the variance of the distribution and I represents the $\mathrm{d} x \mathrm{~d}$ identity matrix. The initial standard deviation $\left(\sigma_{0}\right)$ of the distribution can be computed by Eq. (5).

$$
\sigma_{0}=\frac{\max (\text { upperlimit })-\min (\text { lowerlimit })}{2}
$$

Here, $\sigma_{0}$ can be considered as the initial radius ( $\mathrm{r} 0$ ) of the outer circle for a two-dimensional optimization problem. Because a weak locality is required in the initial phases, $\mathrm{r} 0$ is chosen to be a large value. Ths, a full coverage of the search space by the outer circle is provided in the initial step.

A solution $s^{\prime} \in C_{0}(s)$ is selected and memorized from $C_{0}(s)$ to replace the current circle center $\mu_{0}$. The candidate solutions must be ensured to be inside the search boundaries. The solutions that exceed the boundaries are shifted into the boundaries, as in Eq. (6).

$$
s_{k}^{i}=\left\{\begin{array}{l}
\text { rand. }\left(u l^{i}-l l^{i}\right)+l l^{i}, s_{k}^{i}<l l^{i} \\
s_{k}^{i}, \quad l l^{i} \leq s_{k}^{i} \leq u l^{i} \\
\operatorname{rand} .\left(u l^{i}-l l^{i}\right)+l l^{i}, s_{k}^{i}>u l^{i}
\end{array}\right.
$$

Where $\mathrm{k}=1,2, \ldots, \mathrm{n}$ and $\mathrm{i}=1,2, \ldots, \mathrm{d}$ and rand is a uniformly distributed random number.

To apply the algorithm to TC problem the candidate solution $\overrightarrow{\mathrm{C}_{\mathrm{t}}(\mathrm{s})}$ is converted into a binary vector. Each $\mathrm{C} 0(\mathrm{~s})=\{\mathrm{s} 1, \mathrm{~s} 2, \ldots, \mathrm{sk}\}, \mathrm{k}=1,2, \ldots$, $\mathrm{n}$ takes either the values 1 or 0 which means that the node $\mathrm{i}$ within the final topology is active or inactive, respectively. Eq. (7) is used to convert $\overrightarrow{C_{t}(s)}$ into binary vector (B) using the Sigmoid function $\operatorname{sig}(\mathrm{s})$.

$$
\begin{gathered}
B[i]=\left\{\begin{array}{cr}
1, & \text { if } \operatorname{sig}\left(C_{t}[s]>0.5, \quad i \in[1, k]\right. \\
0, & \text { otherwise }
\end{array}\right. \\
\operatorname{sig}(s)=\frac{1}{1+e^{s}}
\end{gathered}
$$

The random initialization of each position using uniform distribution result in that approximately $50 \%$ of the node will be active this may lead to large convergence time for the algorithm and does not ensure a great diversity of the search agent in the search space. To overcome this problem, we vary the probability of a node being active in the initialization step. Therefore the population is divided into five groups each has $20 \%$ search agents. The groups are initialized with the different percent of initial active nodes: $10 \%, 25 \%, 50 \%, 80 \%$ and $90 \%$, respectively.
Eq. (9) shows the fitness function that is applied to evaluate different solutions. It has two objectives; the first is to minimize the total number of active node in the initial topology, while the second objective is to assure the high residual energy within this active node. The two objectives are to extend the operational lifetime of the wireless network, which will be investigated in future experiments.

$$
f(B)=\sum_{i=1}^{k} \frac{B[i]}{E_{i}}
$$

where $E_{i}$ is the initial energy in node $i$. The equation contributes to the proposed algorithm to focus on the solution with the minimum possible number of active nodes of high residual energy. The dependence on the fitness function alone does not ensure that the final topology will cover all nodes. Therefore a tree traversal algorithm [33] is utilized to check if all nodes are covered by neighbors of active nodes in the topology. The pseudo code of the VSTC algorithm is illustrated in Algorithm 1.

\section{Type-style and fonts}

In this paper, VSTC algorithm was used to optimize the topology control, increasing the remaining lifetime of the network and save the energy consumption for WSN. Due to the nature of the VS algorithm, it is practical to uses a new adaptive step size adjustment scheme that considerably improves the performance of the search process. Then VSTC algorithm was utilized so that it is expected to be more precise for TC construction.

\subsection{Performance evaluation}

Comparing the performance of different algorithms on a given problem is another difficulty that is not as easy as it sounds. In order to calculate the performance of a proposed VSTC algorithm, three algorithms: A1 algorithm, A2 algorithm, and PSOTCS algorithm are studied. The number of nodes $(N), E_{i}$ is the initial energy in node $i$, number of active node (AN), total initial energy (TIE), total energy within the constructed topology for active nodes (TEAN) and the ratio between these active nodes to the residual nodes (RN) are used in evaluating the performance of the compared algorithms as depicted in Eqs. (10) through (12).

$$
\begin{aligned}
& T I E=\sum_{i=1}^{n} E_{i} \\
& T E A N=\sum_{i=1}^{A N} E_{i} \\
& R N=\frac{A N}{n}
\end{aligned}
$$


The energy consumption and lifetime are widely used in performance evaluation for TC and sensor deployment protocol for WSN. In this paper, AN, TIE, TEAN, and RN of the network analysis were used to evaluate the ability of the algorithm to increase the lifetime of the network construction.

Algorithm 1: Pseudo code of VSTC Algorithm.

1. Input: A graph repesent the nodes and their neighbours for WSN.

2. Output: Optimal topology.

3. Generating the initial solution $\mu_{0}$ based on Eq. (1).

4. While Solution < Boundaries do

i. Determined the number of neighbor solutions $C_{t}(s)$.

ii. $\quad$ Foreach $k=1,2, \ldots, n$ do

a. Calculate $C_{0}(s)=\left\{s_{1}, s_{2}, \ldots, s_{k}\right\}$

iii. end

iv. Compute total number of candidate solutions based on Eq. (2).

5. The solutions that exceed the boundaries are shifted into the boundaries based on Eq. (5).

6. Initialize the number of iteration $\mathrm{T}$.

7. While Fitness $>$ Tolerance over $\mathrm{T}$ iterations do

i. Foreach search agent $C_{t}(s)$ do

a. Convert the position into a binary presentation based on Eq. (6)

b. Calculate the agent fitness using Eq. (10). ii. end

8. Obtained the best search agent.

9. Foreach search agent $C_{t}(s)$ do

i. Update $C_{t}(s)$ based on Eq. (4).

10. end

11. Construct the optimial topology based on the best soultion $\overrightarrow{C_{t}(s)}$.

\subsection{Experimental results}

Experimental results obtained in this paper demonstrate that using VSTC algorithm will improve the performance metrics of TC for WSN. In this section, we study the performance of the proposed VSTC model by simulation. For these simulation experiments, we assumed that there are 200 through 1000 sensor nodes (N) distributed in a ten layers circle region. Extensive simulation experiments had been conducted to compare the performance of the proposed VSTC algorithm with the A1, A3 and PSOTCS algorithms. For each network's density, there were a number of 5 different random deployment scenarios within an area of size $1000 \times 1000 \mathrm{~m}$. Each sensor node had a
$100 \mathrm{~m}$, and $20 \mathrm{~m}$ communication and sensing ranges, respectively.

The experiments were conducted to evaluate the quality reducing the number of active nodes that to cover the deployment area and preserves the network's connectivity. Therefore, the performance analysis of the proposed algorithm focuses on a twodimensional matrix; the number of active nodes, and the total energy within the constructed topology of active nodes. Since the results vary due to the uncontrolled random deployments of sensor nodes, the performance analysis calculated the mean of 5 deployment scenarios per each network's density and for each different network size the average number of run (5 runs) of the algorithm is calculated for all the compared algorithms A3, A1, PSOTCS and the VSTC. Table 1, illustrates that VSTC algorithm was achieved a reduced topology and decreased the number of AN by 18\%,12\% and 10\% using the VSTC algorithm compared with A1, A3, PSOTCS respectively.

Fig. 3 shows the number of $\mathrm{AN}$, is linearly increased with the growth of network's size, however, the number of active nodes cannot be considered as a ratio to the network's density.

Fig. 4 illustrates the ratio between these active nodes to the RN vs N, VSTC has achieved the best results compared with the existing algorithms. It is not only substantial to reduce the number of active nodes, whereas it is significant to do the selected nodes possess enough residual energies. To do that, the active nodes do its function well as much as possible considering they are the bridge between the sink node and other nodes. As in the case of selecting a low residual energy node to join the active topology, its energy is to deplete quickly, and then the topology will be influenced. This rule had been considered in the VSTC algorithm through the application of the proposed fitness function. Fig. 5 shows the TEAN vs TIE. As observed from Fig. 6, VSTC algorithm has obtained the best results through various network sizes and save the residual energies.

Fig. 6 shows that the proposed VSTC algorithm achieved nearly $34 \%$ higher energies levels within the constructed topology than the A1. As observed from Figs. 5 and 6, VSTC algorithm has obtained the best results through various network sizes and save the residual energies.

\subsection{Summary of remark}

As an observation from Table 2, it is seen that the proposed VSTC algorithm has achieved the best 
Table 1. Experimental results for A1, A3, PSOTCS and VSTC algorithms

\begin{tabular}{|c|c|c|c|c|c|c|}
\hline \multirow{2}{*}{ Alg. } & \multirow{2}{*}{ Metrics } & \multicolumn{5}{|c|}{ Network Size } \\
\hline & & 200 & 400 & 600 & 800 & 1000 \\
\hline \multirow{4}{*}{ A1 } & TIE & 202298 & 401229 & 602072 & 798082 & 1006281 \\
\hline & AN & 37 & 39 & 43 & 43 & 45 \\
\hline & TEAN & 34560 & 36780 & 37890 & 38870 & 40060 \\
\hline & RN & 0.185 & 0.098 & 0.072 & 0.054 & 0.045 \\
\hline \multirow{4}{*}{ A3 } & TIE & 202298 & 401229 & 602072 & 798082 & 1006281 \\
\hline & AN & 35 & 38 & 40 & 39 & 42 \\
\hline & TEAN & 36460 & 37982 & 39386 & 40978 & 43113 \\
\hline & RN & 0.175 & 0.095 & 0.067 & 0.049 & 0.042 \\
\hline \multirow{4}{*}{ PSOTCS } & TIE & 202298 & 401229 & 602072 & 798082 & 1006281 \\
\hline & $\mathrm{AN}$ & 33 & 35 & 37 & 38 & 41 \\
\hline & TEAN & 40790 & 41230 & 42568 & 44328 & 46580 \\
\hline & RN & 0.165 & 0.088 & 0.062 & 0.048 & 0.041 \\
\hline \multirow{4}{*}{ VSTC } & TIE & 202298 & 401229 & 602072 & 798082 & 1006281 \\
\hline & $\mathrm{AN}$ & 28 & 32 & 33 & 35 & 37 \\
\hline & TEAN & 44657 & 48980 & 50456 & 55236 & 60454 \\
\hline & RN & 0.140 & 0.080 & 0.055 & 0.044 & 0.037 \\
\hline
\end{tabular}

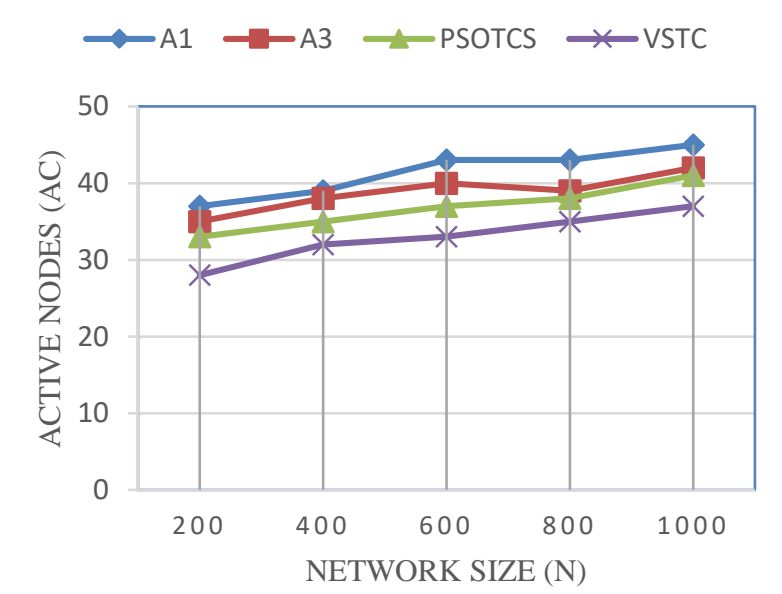

Figure.3 AN of the network for varying of network size (N)

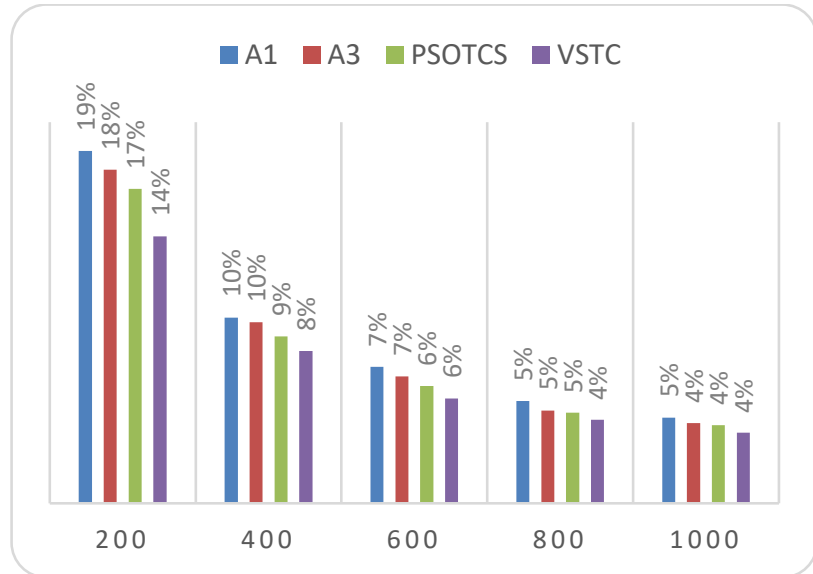

Figure.4 RN for varying of network size $(\mathrm{N})$

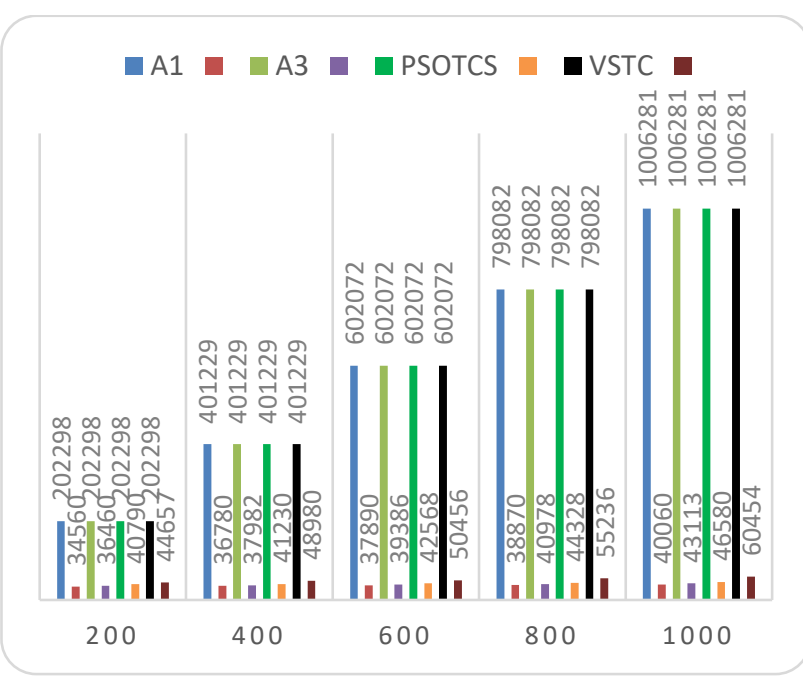

Figure.5 TEAN vs TIE

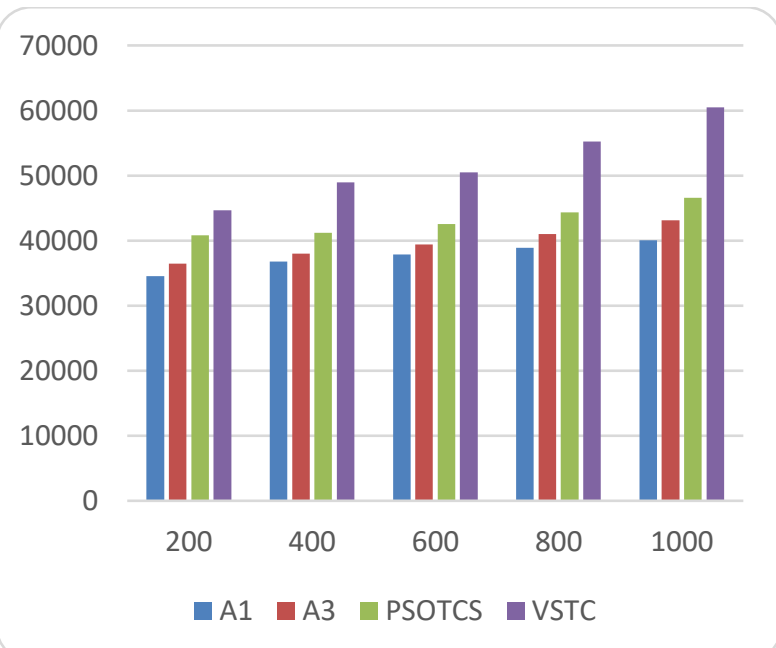

Figure.6 TEAN for varying of network size $(\mathrm{N})$ 
Table 2. Comparision of the maximum performance evaluation obtained for various algorithms.

\begin{tabular}{lll}
\hline Algorithms & AN & TEAN \\
\hline A1 & 45 & 40060 \\
A3 & 42 & 43113 \\
PSOTCS & 41 & 46580 \\
Proposed VSTC & 37 & 60454 \\
\hline
\end{tabular}

results and provides $18 \%$ and $34 \%$ in terms of $\mathrm{AN}$ and TEAN respectively in comparison to A1 algorithm, $12 \%$ and $29 \%$ in terms of AN and TEAN respectively in comparison to the recent the A3 algorithm, and $10 \%$ and $23 \%$ in terms of $\mathrm{AN}$ and TEAN respectively in comparison to the PSOTCS algorithm.

\section{Conclusion}

The goal of this paper is to present a TC algorithm based on vortex search algorithm termed VSTC algorithm in WSNs. Moreover, the proposed algorithm introduces a function that minimizes the number of active nodes while assuring the resident high energies within the selected nodes without losing the network coverage and connectivity features. Furthermore, the performance analysis of the proposed algorithm, the A1 topology algorithm, the A3 topology algorithm, and PSOTCS algorithm are demonstrated. The experimental results showed that the advantages of the proposed algorithm in terms of a considerable reduction in the number of active nodes while maintaining a high-energy range within the constructed topology compared with the existing algorithms. Our future research will focus on implementing genetic algorithm, machine learning techniques and other swarm intelligence algorithms in terms of similar methods presented in the paper. We will also try to construct a fault tolerant network topology.

\section{References}

[1] M. Zungeru, A. Li-Minn, and P. S. Kah "Classical and swarm intelligence based routing protocols for wireless sensor networks: A survey and comparison", Journal of Network and Computer Applications, Vol. 35, No. 5, pp.1508-1536, 2012.

[2] Y. Yao and J. Nanlan, "Distributed wireless sensor network localization based on weighted search", Computer Networks, Vol.86, pp.57-75, 2015.

[3] J. Ben-Othman, B. Karim, B. Alain, and P. Laurence, "Self-stabilizing algorithm for efficient topology control in wireless sensor networks", Journal of Computational Science, Vol. 4, No. 4, pp.199-208, 2013.

[4] T. Laukkarinen, S. Jukka, and H. Marko, "A survey of wireless sensor network abstraction for application development", International Journal of Distributed Sensor Networks, Vol. 8, No. 12 pp.740268, 2012.

[5] M. Saleem, A. D. Gianni, and F. Muddassar, "Swarm intelligence based routing protocol for wireless sensor networks: Survey and future directions", Information Sciences, Vol. 181, No. 20, pp.4597-4624, 2011.

[6] G. Mao, F. Barış, and D. Brian, "Wireless sensor network localization techniques", Computer Networks, Vol. 51, No. 10, pp.2529. 2553, 2007.

[7] R. Wattenhofer, L. Li, B. Paramvir, and W. YM., "Distributed topology control for power efficient operation in multihop wireless ad hoc networks", In: Proc. of the twentieth annual joint conference of the IEEE computer and communications societies, Vol. 3, pp. 13881397, 2001.

[8] L. LoBello and T. Emanuele, "An adaptive approach to topology management in large and dense real-time wireless sensor networks", IEEE Transactions on Industrial Informatics, Vol. 5, No. 3, pp.314-324, 2009.

[9] E. H. Houssein, and A. A. Ismaeel, "Ant Colony Optimization based Hybrid Routing Protocol for MANETs", Journal of Emerging Trends in Computing and Information Sciences, Vol. 6, No. 11, pp. 622-629, 2015.

[10] Doğan, and Ö. Tamer, "A new metaheuristic for numerical function optimization: Vortex Search algorithm", Information Sciences, Vol. 293, pp.125-145, 2015.

[11] S. Rizvi, H. K. Qureshi, S. A. Khayam, V. Rakocevic, and M. Rajarajan, "A1: An energy efficient topology control algorithm for connected area coverage in wireless sensor networks", Journal of Network and Computer Applications, Vol. 35, No. 2, pp.597-605, 2012.

[12] P. M. Wightman, and A. L. Miguel, "A3: A topology construction algorithm for wireless sensor networks", In: Proc. of the Global Telecommunications Conference, IEEE GLOBECOM 2008, pp. 1-6, 2008.

[13] Y. Bingyu, G. Chen, and W. Guo, "Topology control in wireless sensor networks based on discrete particle swarm optimization", In: Proc. of the IEEE International Conference on Intelligent Computing and Intelligent Systems, Vol. 1, pp. 269-273, 2009. 
[14] W. Liao, K. Yucheng, and L. Ying-Shan, "A sensor deployment approach using glowworm swarm optimization algorithm in wireless sensor networks", Expert Systems with Applications, Vol. 38, No. 10, pp.2180-12188, 2011.

[15] R. C. Abreu and E. C. A. José, "A Particle Swarm Optimization Algorithm for Topology Control in Wireless Sensor Networks", In: Proc. of the 30th International Conference of the Chilean Computer Science Society, pp.8-13, 2011.

[16] A. Nikdel, M. Mahdi, and N. Hagar, "An Intelligent PSO-based Topology Control Protocol for Wireless Sensor Networks", International Journal of Smart Home, Vol. 8, No. 4, pp.123-138, 2014.

[17] W. Guo, Z. Bin, C. Guolong, W. Xiaofeng, and $\mathrm{X}$. Naixue, "A PSO-Optimized minimum spanning tree-based topology control scheme for wireless sensor networks", International Journal of Distributed Sensor Networks, 2013.

[18] L. Guo, W. Jiqiang, R. Meirui, Z. Zhongzhao, and Y. Yan, "A PSO-based topology control algorithm in wireless sensor networks", In: Proc. of the 5th International Conference on Wireless Communications, Networking and Mobile Computing. IEEE, 2009.

[19] S. Goyal and S. P. Manjeet, "Modified Bat Algorithm for Localization of Wireless Sensor Network", Wireless Personal Communications, Vol. 86, No.2, pp.657-670, 2016.

[20] B. Zhang, J. Zhenzhen, L. Cheng, Y. Zheng, and V. V. Athanasios, "Efficient location based topology control algorithms for wireless ad hoc and sensor networks", Wireless Communications and Mobile Computing, Vol. 16, No. 1, pp.1943-1955, 2016.

[21] N. Ababneh, "Performance evaluation of a topology control algorithm for wireless sensor networks", International Journal of Distributed Sensor Networks, Vol. 6, No. 1, pp. 671385, 2010.

[22] S. Ullah and W. Mussarat, "Topology Control of wireless sensor network using Quantum Inspired Genetic algorithm", arXiv preprint arXiv: pp.1508.02521, 2015.

[23] G. Aloor and J. Lillykutty, "Distributed wireless sensor network localization using stochastic proximity embedding", Computer Communications, Vol. 33, No..6, pp.745-755, 2010.

[24] Y. Wang, "Topology control for wireless sensor networks", Wireless sensor networks and applications, pp. 113-147, 2008.
[25] M. Li, L. Zhenjiang, and V. V. Athanasios, "A survey on topology control in wireless sensor networks: Taxonomy, comparative study, and open issues", In: Proc. of the IEEE, Vol. 101, No.12, pp.2538-2557, 2013.

[26] N. Li, C. H. Jennifer, and S. Lui, "Design and analysis of an MST-based topology control algorithm", IEEE Transactions on Wireless Communications, Vol. 4, No.3, pp.1195-1206, 2005.

[27] Y. Wang, H. Chun-Chi Hu, and T. Yu-Chee, "Efficient deployment algorithms for ensuring coverage and connectivity of wireless sensor networks", In: Proc. of the First International Conference on Wireless Internet, IEEE, pp. 114-121, 2005.

[28] S. Poduri and S. S. Gaurav, "Constrained coverage for mobile sensor networks", In: Proc. of IEEE International Conference on Robotics and Automation, Vol. 1, pp. 165-171, 2004.

[29] Y. Zou and C. Krishnendu, "Sensor deployment and target localization based on virtual forces", In: Proc. of the Twenty-Second Annual Joint Conference of the IEEE Computer and Communications. IEEE Societies, Vol. 2, pp.1293-1303, 2003.

[30] A.P. Renold and S. Chandrakala, "Survey on state scheduling-based topology control in unattended wireless sensor networks", Computers \& Electrical Engineering, Vol.56, pp.334-349, 2016.

[31] P. Santi, "Topology control in wireless ad hoc and sensor networks", ACM computing surveys (CSUR), Vol. 37, No.2, pp.164-194, 2005.

[32] M. A. Khan, K. Asfandyar, K. S. Said, and A. Azween, "An Energy Efficient Color Based Topology Control Algorithm for Wireless Sensor Networks", Wireless Sensor Network, Vol.5, No. 1, p.1-7, 2013.

[33] N. C Kalra and P. C. P. Bhatt, "Parallel algorithms for tree traversals", Parallel Computing, Vol. 2, No.2, pp.163-171, 1985. 